\title{
Health Care Availability asa Fundamental Predictor of Chronic Kidney Diseases
}

\author{
Gennadii S Petrov ${ }^{1}$, Irina L Krom ${ }^{1,2 *}$ and Marina V Yerugina ${ }^{1}$ \\ ${ }^{1}$ Department of Health Organization, Fsbei He Vi Razumovsky Saratov, Russia \\ ${ }^{2}$ Department of Health Organization, Centre of Medical-Sociological Research, Russia
}

*Corresponding author: Irina L Krom, Department of Health Organization and Public Health with the Courses of Law and History of Medicine, Centre of Medical-Sociological Research, Saratov, Russia

Submission: 眥July 05, 2018; Published: 監July, 17, 2018

\begin{abstract}
Chronic kidney diseases are recognized as one of the leading problems of modern healthcare because of the high prevalence and unfavorable prognosis. Modern classification of predictors of chronic kidney diseases are discussed in the article. The authors of the article note that none of the known modern classifications of chronic kidney diseases consider medical care availability to patients in disease situations as a predictor. The authors worked out typology of availability of nephrological medical care to chronic kidney disease patients is given in the article. Medical care availability restriction to patients is considered by the authors as an obligate predictor of the occurrence, progression and unfavorable prognosis of chronic kidney diseases.

Keywords: Chronic kidney diseases; Predictors; Availability of medical care; Chronic kidney; Prevalence; Predictors; Nephrology; Patients; Population; Biochemical; Geographic; Economic; Social; Barriers; Occurrence; Federation; Microalbumin; Serum; Blood; Transplantation; Hemodialysis; Therapy; Restriction
\end{abstract}

\section{Introduction}

According to international population studies [1,2], 10-14\% of the world's population is in pain from chronic kidney diseases (CKD). Chronic kidney diseases are recognized as one of the leading problems of modern healthcare because of the high prevalence and unfavorable prognosis.The following current classifications of CKD predictors are known.In 2005 AS Levey [3] and co-authors proposed classification of the main predictors of CKD, dividing them into predictors, which increase susceptibility, predictors of initiation, progression and terminal stage of CKD.

G Barkis [4] divided the factors of progression into traditional and "new", most of which are biochemical markers of CKD progression, most often directly involved in the pathogenetic mechanisms of progression.

The classification of predictors published in the 2012 national guidelines is based on the division of predictors of risk development and progression [5]. The principal difference of this classification lies in the division of predictors to modifiable and non-modifiable, which is of great practical importance.However, none of the known modern classifications does not consider medical care availability in the situation of a disease as a predictor of CKD. Health care availability is a free access to health resources regardless of geographic, economic, social, cultural, organizational or language barriers [6].

The typologization of nephrological medical care availability to patients with CKD was developed by G.S. Petrov and co-authors:

a. Availability of prevention and minimization of CKD predictors occurrence.

b. Availability of modifiable predictors management of CKD progression and nephroprotection.

C. Availability of substitution renal therapy for patients with CKD.

Availability of prevention and minimization of CKD predictors occurrence.At present time, there is a program in Russia for the prophylactic medical examination of the population, which is regulated by the Order № 1006 of the Ministry of Health of the Russian Federation dated December, 3rd 2012 "On the approval of the procedure for the prophylactic medical examination of certain groups of adults" [6]. Main CKD markers- serum blood creatinine and the determination of microalbumin in the urine are simple and fairly inexpensive tests, but they are not included in this program. The insertion of serum blood creatinine and microalbuminuria 
into the program for the prophylactic medical examination of the population is one of the important tasks of preventing CKD.

The CKD prevention requires regular monitoring of patients with the above mentioned conditions by thetherapist, and, in case of detecting CKD predictors, the refer and examination of the nephrologist.Availability of management of modifiable predictors of CKD progression andnephroprotection.In case of CKD the necessity to manage the modifiable risk factors of CKD progression and nephroprotection arisesto prevent the progression of the disease. Nephroprotective strategy is based both on drug therapy aimedat saving the remaining functioning nephrons, and on the correction of other risk factors of nephrondamage, such as lifestyle, treatment of the underlying nephrologic disease, etc.

Availability of substitution renal therapy for patients with CKD Substitution therapy to patients with CKDis indicated in case of futility of nephroprotective therapy and in the terminal phase of the disease.

There exist 3 types of substitution therapy for patients with CKD
a. Hemodialysis.
b. Peritoneal dialysis.
c. Transplantation of the kidney.

\section{Conclusion}

According to [7], in 2010, 2,6 million patients in the world were on dialysis treatment, $93 \%$ of them were residents of countries with high- and middle-income level. However, according to the authors' calculations based on the prevalence of CKD, the total number of patients requiring renal substitution therapy may vary between 4,9 and 9 million people. The above calculations make it possible to suggest that at least 2,3 million patients are unable to receive renal substitution therapy in a timely manner, which inevitably increases the adverse result.

Thus, the availability of nephrological care for patients is realized in the availability of prevention and minimization of predictors for the occurrence of CKD, in the management of modifiable predictors of CKD progression and nephroprotection, and renal substitution therapy for patients with CKD. Medical care availability restriction to patients is considered as an obligate predictor of the occurrence, progression and unfavorable prognosis of chronic kidney diseases.

\section{References}

1. Imai E, Horio M, Iseki K, Yamagata K, Watanabe T, at el. (2007) Prevalence of chronic kidney disease (CKD) in the Japanese general population predicted by the MDRD equation modified by a Japanese coefficient. Clin exp nephrol11(2): 156-163.

2. Singh AK, Farag Youssef MK, Mittal BV, Subramanian KK, Reddy SRK, et al. (2013) Epidemiology and risk factors of chronic kidney disease in India - results from the SEEK (Screening and Early Evaluation of Kidney Disease) study. BMC Nephrol 14(1): 114.

3. Levey AS, Eckardt KU, Tsukamoto Y, Levin A, Coresh J,et al. (2005) Definition and classification of chronic kidney disease: a position statement from Kidney Disease: Improving Global Outcomes (KDIGO). Kidney International 67(6): 2089-20100.

4. Barkis GL (2010) Chronic kidney disease and hypertension. Satellite Symposium "Control of cardiovascular risk with cardiometabolic syndrome patients in the framework of the ISH-2010 congress. Vancouver.

5. Smirnov AV, Schilov EM, Dobronravov VA, Kayukov IG, Bobkova IN, et al. (2012)National recommendations. Chronic illness of kidneys: main principles of screening, diagnostics, preventive maintenance and approaches to treatment. Clinical Nephrology (4): 4-26.

6. (2015) Health and human rights (WHO) Fact sheet.

7. GarciaGG, Jha V, Tao Li PK, Couser WG, Erk T, et al. (2015) CKD in Disadvantaged Populations. Clin kidney J 8(1): 3-6.
Creative Commons Attribution 4.0 International License

For possible submissions Click Here
Submit Article

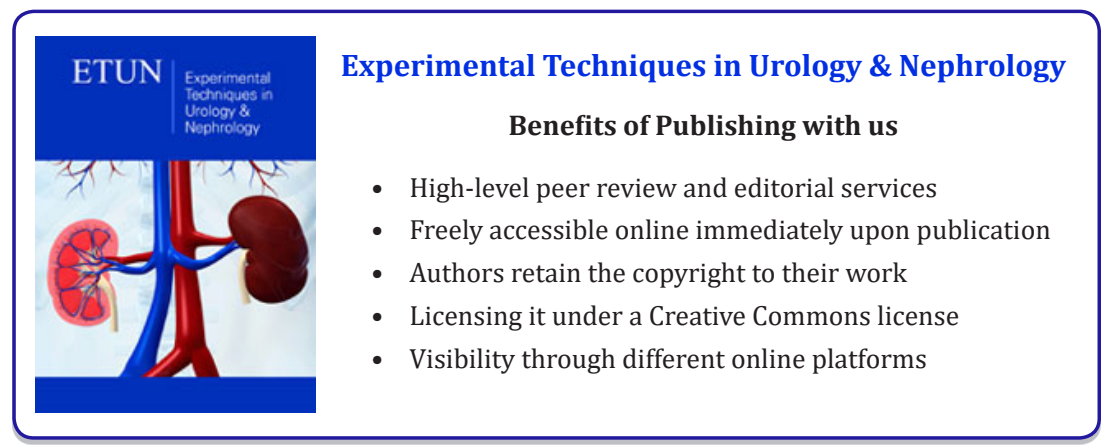

